\title{
Oxygen Tolerant PET-RAFT Facilitated 3D Printing of Polymeric Materials under Visible LEDs
}

Ali Bagheri ${ }^{\mathrm{a}, \mathrm{b}^{*}}$, Chris William Anderson Bainbridge ${ }^{\mathrm{a}}$, Kyle Edward Engel ${ }^{\mathrm{a}}$, Greg G. Qiao c, Jiangtao Xu ${ }^{\mathrm{d}}$, Cyrille Boyer ${ }^{\mathrm{d}}$, and Jianyong Jin ${ }^{\mathrm{a}, \mathrm{b}^{*}}$

a School of Chemical Sciences, The University of Auckland, Auckland 1010, New Zealand

${ }^{\mathrm{b}}$ Dodd-Walls Centre for Quantum and Photonic Technologies, Auckland 1010, New Zealand

${ }^{\mathrm{c}}$ Department of Chemical Engineering, The University of Melbourne, Parkville, Victoria 3010, Australia ${ }^{d}$ Centre for Advanced Macromolecular Design (CAMD) and Australian Centre for NanoMedicine (ACN), School of Chemical Engineering, The University of New South Wales, Sydney NSW 2052, Australia

*Email: ali.bagheri@auckland.ac.nz

*Email: j.jin@auckland.ac.nz 


\section{Materials}

Poly (ethylene glycol) diacrylate (PEGDA 250) (average $M_{n} 250 \mathrm{~g} / \mathrm{mol}$ ) and $n$-butyl acrylate (BA) were purchased from Sigma Aldrich and deinhibited by passing through a column of basic alumina. Poly (ethylene glycol) diacrylate (PEGDA 6000) (average $M_{n} 6000 \mathrm{~g} / \mathrm{mol}$ ), dibenzyl trithiocarbonate (DBTTC), 4-cyano-4-[(dodecylsulfanylthiocarbonyl) sulfanyl] pentanoic acid (CDTPA), triethylamine (TEA) and eosin Y (EY) were purchased from Sigma Aldrich and used as received. All the other reagents were used as received unless otherwise specified.

\section{Characterization}

Scanning Electron Microscopy (SEM) was performed using a FEI Quanta 200 field emission environmental SEM. A scanning voltage of $10 \mathrm{KeV}$ and a spot size of 3 was used. Samples were characterized with a working distance of $11-13.5 \mathrm{~mm}$. A low vacuum secondary electron detector was used.

Photo Differential Scanning Calorimetry (photo-DSC) was performed using a commercial Netzsch PhotoDSC consists of a DSC3500 Sirius unit coupled with Klerex Delolux 04 light source (400-500 nm). Samples were placed into an open top aluminium pan and the exposure time was set at $16 \mathrm{~min}$. Samples were measured under isothermal conditions at $25^{\circ} \mathrm{C}$ and a nitrogen purge flow of $250 \mathrm{ml} / \mathrm{min}$.

The contact angle (CA) measurement was performed using a Contact Angle Meter (CAM) 100 by dispensing a water droplet with a threaded micro syringe. The optical images of the water contact angle were recorded and analysed by using the CAM 100 software. The program uses a Young-Laplace equation to calculate the drop shape. The values of the parameters were repeated until an acceptable compromise between the actual drop profile and the model was achieved. The measurements were performed at ambient room temperature and pressure.

A modified ANYCUBIC Photon DLP 3D printer was used for 3D printing of RAFT-based resin formulations, where visible LED lights (blue $\left(\lambda_{\max }=483 \mathrm{~nm}, 4.16 \mathrm{~mW} / \mathrm{cm}^{2}\right)$ or green $\left(\lambda_{\max }=532 \mathrm{~nm}\right.$, $\left.0.48 \mathrm{~mW} / \mathrm{cm}^{2}\right)$ ) were focused on the bottom surface of the resin vat. Photo-curing occurs between the bottom of the vat and the motorized build platform through an in-house built mask. 3D printing parameters such as layer exposure times and layer thickness were set using ANYCUBIC Photon Slicer software. The 
3D models were designed using an online software provided by Autodesk ${ }^{\circledR}$ Tinkercad ${ }^{\mathrm{TM}}$, which were then transferred to the slicing software.

Emission spectra of the light sources used were obtained using a Schoeffel Instruments monochromator coupled to a Newport 1815-C power meter and 818-SL detector. The monochromator was scanned through the light spectrum using a diffraction grating connected to a micrometer gauge. The power of each selected wavelength is then measured by the optical meter, and lastly converted from power $(\mathrm{mW})$ to intensity $(\mathrm{mW} / \mathrm{cm} 2)$ by dividing each value by the detector area.

Penetration depth measurements were performed by allowing the light source to shine through a photomask into a PET-RAFT resin for a period of 12 hours. The height of the solid object was measured using digital calipers and taken to be the penetration depth at that wavelength.

Swelling ratios of networks were measured in fully swollen state at room temperature: swelling ratio defined as $W_{w} / W_{d}$, where $W_{w}$ is the weight of a sample swollen in DMSO; $W_{d}$ is the weight of sample in the dry state.

\section{EXPERIMENTAL SECTION}

General Procedure for PET-RAFT Facilitated 3D Printing using Visible Light-Curable RAFT-Based Resin Formulations;

A PET-RAFT resin formulation using a molar ratio of $[$ PEGDA]/[CDTPA]/[EY]/[TEA] = 200:1:0.04:3 was set up as follows: a solution containing PEGDA $250(10.0 \mathrm{ml}, 44.8 \mathrm{mmol})$, EY (5.8 mg, 0.00896 mmol), TEA (93.7 $\mu \mathrm{l}, 0.672 \mathrm{mmol})$, and CDTPA (90.4 mg, $0.224 \mathrm{mmol}$ ) was prepared. The solution was mixed and sonicated until all the components were dissolved.

A PET-RAFT resin formulation using a molar ratio of [PEGDA]/[CDTPA]/[EY]/[TEA] = 50:1:0.01:3 was set up as follows: a solution containing PEGDA 250 (6.0 ml, $26.88 \mathrm{mmol})$, EY (3.5 mg, $0.00538 \mathrm{mmol})$, TEA $(224.8 \mu \mathrm{l}, 1.61 \mathrm{mmol})$, and CDTPA $(217.0 \mathrm{mg}, 0.538 \mathrm{mmol})$ was prepared. The solution was mixed and sonicated until all the components had dissolved. 
A PET-RAFT resin formulation using a molar ratio of [PEGDA 250]: [PEGDA 6000]: [BA]: [DBTTC]:[EY]:[TEA] = 45:0.04:4:1:0.01:3 was set up as follows: a solution containing PEGDA 250 (3.0 ml, $13.44 \mathrm{mmol}$ ), PEGDA 6000 (78.0 mg, $0.013 \mathrm{mmol})$, BA (153.0 mg, $1.19 \mathrm{mmol})$, EY (1.9 mg, 0.00299 mmol), TEA (124.8 $\mu 1,0.896 \mathrm{mmol})$, and DBTTC ( $86.8 \mathrm{mg}, 0.299 \mathrm{mmol})$ was prepared. The solution was mixed and sonicated until all the components had dissolved. This formulation was used in the experiments showed in Figure 5.

\section{Preparation of Sample Resins for Photo-DSC Analysis}

For photo-DSC analysis, samples with independently varying concentrations of [PEGDA 250] or [TEA] or $[\mathrm{EY}]$ were produced to monitor the effect of component concentration on the reaction kinetics.

-An example DSC sample for varying [PEGDA 250] was prepared as follows: $([\mathrm{PEGDA}] /[\mathrm{CDTPA}] /[\mathrm{EY}] /[\mathrm{TEA}]=(1000-50): 1: 100(\mathrm{ppm}): 1) ;$ e.g.

PEGDA 250 (61.9 mg, $0.2477 \mathrm{mmol})$, CDTPA (2.0 mg, $0.00495 \mathrm{mmol})$, EY (32.0 $\mu 1$ from $1 \mathrm{mg} / \mathrm{ml}$ stock solution, $\left.4.96 \times 10^{5} \mathrm{mmol}\right)$, and TEA $(1.4 \mu 1,0.00991 \mathrm{mmol})$ were mixed in a vial until all components had dissolved.

-An example DSC sample for varying [TEA] was prepared as follows: ([PEGDA]/[CDTPA]/[EY]/[TEA] $=200: 1: 0.04:(0.2-4)$; e.g.

TEA (1.38 $\mu 1,0.00991 \mathrm{mmol})$, CDTPA $(2.0 \mathrm{mg}, 0.00495 \mathrm{mmol})$, EY (128.4 $\mu 1$ from $1 \mathrm{mg} / \mathrm{ml}$ stock solution, $\left.1.98 \times 10^{4} \mathrm{mmol}\right)$, and PEGDA $250(247.7 \mathrm{mg}, 0.991 \mathrm{mmol})$ were mixed in a vial until all components had dissolved.

-An example DSC sample for varying [EY] was prepared as follows: ([PEGDA]/[CDTPA]/[EY]/[TEA] = 200:1:(10-400 ppm):2; e.g.

EY (128.4 $\mu \mathrm{L}$ from $1 \mathrm{mg} / \mathrm{ml}$ stock solution, $\left.1.98 \times 10^{4} \mathrm{mmol}\right)$, CDTPA (2.0 mg, $\left.0.00495 \mathrm{mmol}\right)$, PEGDA $250(247.7 \mathrm{mg}, 0.991 \mathrm{mmol})$, and TEA $(1.4 \mu 1,0.00991 \mathrm{mmol})$ were mixed in a vial until all components had dissolved.

\section{Post-Printing Modification of the 3D Printed Objects}


A growth medium containing BA $(63.0 \mathrm{mg}, 0.4912 \mathrm{mmol})$, and DMSO $(98.2 \mu \mathrm{l})$ was prepared. The parent 3D printed object ([PEGDA 250]:[PEGDA 6000]:[BA]:[DBTTC]:[EY]:[TEA] = 45:0.04:4:1:0.01:3) was then soaked in this solution for $24 \mathrm{~h}$ and degassed for 20 minutes at room temperature, before being irradiated under a blue LED light $\left(\lambda_{\max }=460 \mathrm{~nm}, 0.7 \mathrm{~mW} / \mathrm{cm}^{2}\right)$ for $6 \mathrm{~h}$. After the polymerization, the modified object was removed and was extensively washed to remove unreacted BA monomers, before being taken for contact angle analysis (results showed in Figure 5).

Results reported in the Figure $\mathbf{S 2}$ were obtained using:

A growth medium containing BA (126.0 mg, $0.9824 \mathrm{mmol})$, and DMSO (196.4 $\mu \mathrm{L})$ was prepared. Parent $3 \mathrm{D}$ printed objects (using formulation with the following ratio: [PEGDA]/[DBTTC]/[EY]/[TEA] = 50:1:0.01:3) were soaked in this solution for $12 \mathrm{~h}$ and degassed for 30 minutes at room temperature, before being irradiated under a blue LED light $\left(\lambda_{\max }=460 \mathrm{~nm}, 0.7 \mathrm{~mW} / \mathrm{cm}^{2}\right)$ for a predefined length of time. After the polymerization, the modified object was removed and was extensively washed to remove unreacted BA monomers, before being taken for contact angle analysis. 


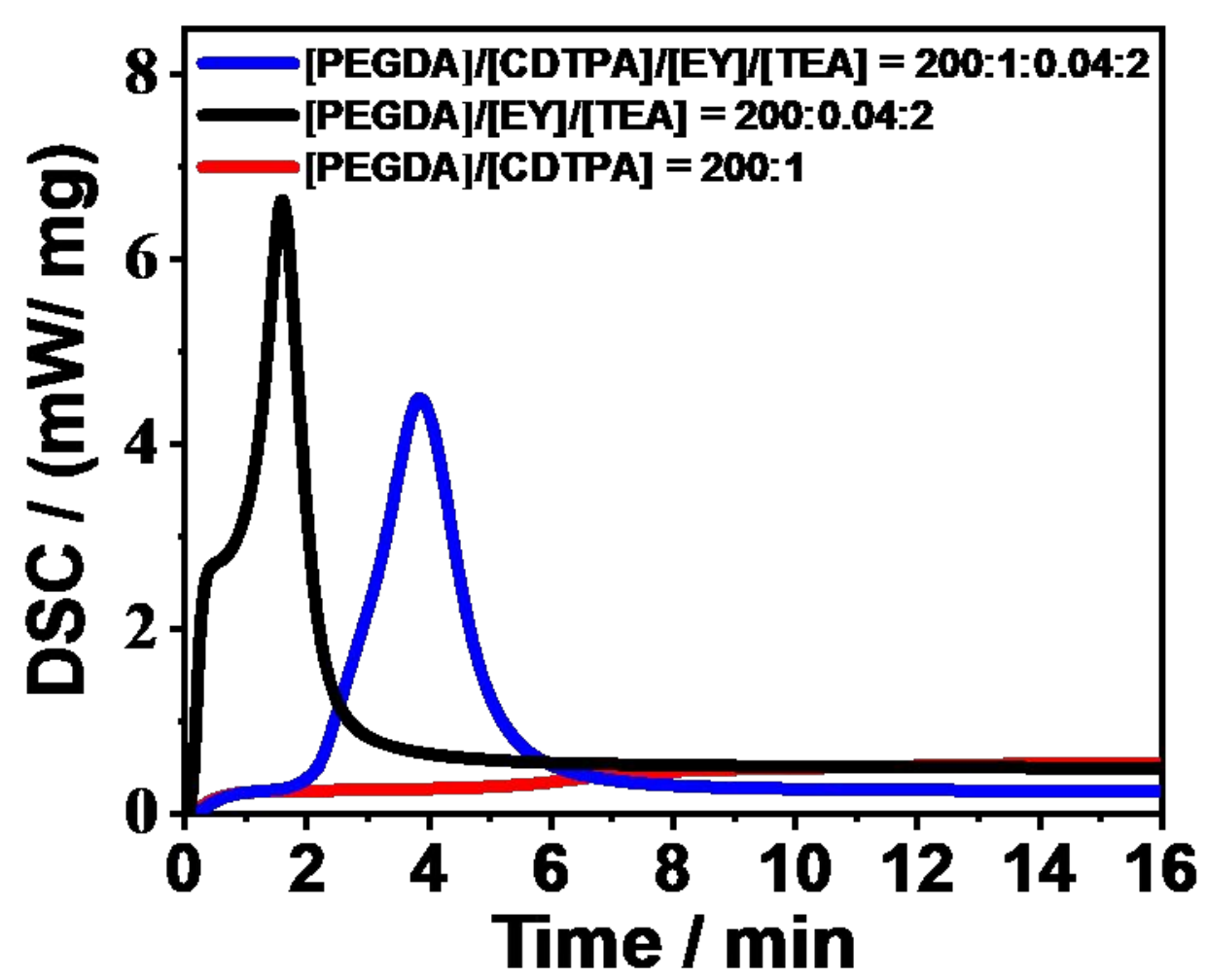

Figure S1. Comparison between photo-DSC signals of PET-based resin, iniferter-based formulations and free radical photopolymerization ([PEGDA]/[EY]/[TEA] $=200: 0.04: 2$ ). 
a) $50.1^{\circ}$

b) $84.5^{\circ}$

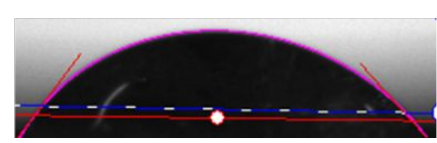

c) $96.5^{\circ}$

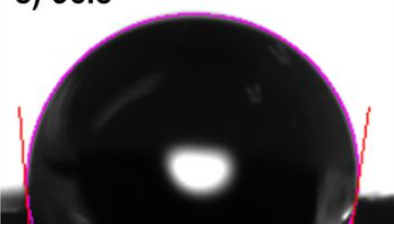

d) $112.6^{\circ}$

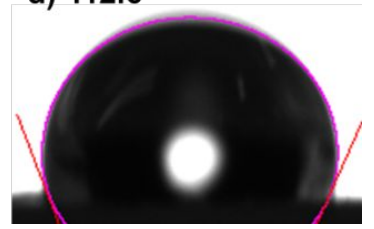

Figure S2. (a) Water contact angles of an initially printed object $\left(50.1^{\circ}\right)$, and its subsequent modified network after BA insertion after (b) 4h, (c) 10h and (d) 18h light exposure. Polymerization of BA or in another word, BA monomer insertion into the printed objects was performed under blue LED light $\left(\lambda_{\max }=460 \mathrm{~nm}, 0.7 \mathrm{~mW} / \mathrm{cm}^{2}\right)$, without presence of external initiators or catalysts. 